\section{Nanotube sensors}

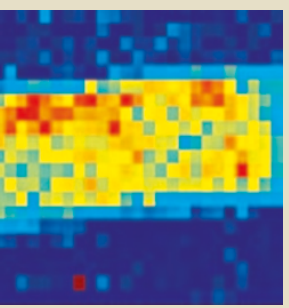

Carbon nanotubes could potentially serve as biosensors, as they emit fluorescence in the near infrared, a region of the spectrum not occupied by other organic molecules and where tissue is transparent. However, applying functionality to nanotubes without destroying their integrity or optical properties has been problematic. Now Stano and colleagues describe a method for adding ligands noncovalently to nanotubes, which leaves the structures intact. They first coated the surface of the nanotube with a monolayer of glucose oxidase, and functionalized the surface by adding an electron acceptor (ferricyanide), which attaches to the surface of the nanotube between the enzyme molecules. In the presence of glucose, the enzyme creates glucolactone and hydrogen peroxide, which shuttles electrons to ferricyanide, causing a shift in emission proportional to the concentration of glucose. By inserting a capillary tube containing such functionalized nanotubes into human tissue, they could show that the nanotube senses glucose concentration in the range found in the blood of diabetics (1-8 $\mathrm{mM}$ ). The authors point out that other functionalities could be applied to nanotubes.

(Nat. Mat. 4, 86-92, 2005)

\section{Novel antibiotic for tuberculosis}

Tuberculosis, after AIDS, is the second most deadly infectious disease, killing millions of people worldwide. Although the number of tuberculosis cases is increasing and drug-resistant strains are continuously emerging, new tuberculosis-specific drugs have not been developed in the past 40 years. New drugs would ideally work within shorter time frames and with less frequent dosing regimens to help overcome bacterial resistance. Now, a team of scientists has identified a new tuberculosis drug candidate, R207910, belonging to the diarylquinoline class of compounds that inhibits both drug-sensitive and drug-resistant strains of Mycobacterium tuberculosis in vitro. R207910 was also found to be more effective, with accelerated bactericidal activity, in vivo, when it replaced one of the drugs making up a tuberculosis treatment cocktail. Unlike existing tuberculosis drugs, R207910 was found to work on a new target, inhibiting the mycobacterium's proton pump ATP synthase. What's more, R207910 was tolerated in healthy human volunteers at plasma level concentrations greater than the optimal effective dose in mice. (Science Express; published online 9 December 2004, doi10.1126/ science.1106753)

\section{Protease substrates for tumor targeting}

Specific targeting of tumor cells in vivo remains a key challenge for molecular imaging applications and therapeutic strategies. Tsien and colleagues now introduce an approach for targeting tumor cells that takes advantage of the excretion of particular proteases by different

Research Highlights written by Kathy Aschheim, Nadia Cervoni, Laura DeFrancesco, Teresa Moogan and Gaspar Taroncher-Oldenburg. tumor cells. The authors first bind a cargo of interest, in this case a fluorescent protein, to a so-called cell-penetrating peptide (CPP), a well-characterized class of sequences that mediates the translocation of covalently attached payloads across mammalian cell membranes. The technological twist that makes these CPPs specific to a target cell consists of fusing the CPP to a negatively charged inhibitory domain by a peptide linker containing a protease-specific sequence. This prevents the CPP and its cargo from entering cells under normal conditions but, in the presence of a secreted protease that cleaves the linker peptide sequence, 'activates' the CPP and its cargo for penetrating cells in its immediacy. Using this approach, the authors image human tumor cells secreting matrix metalloproteinases 2 and 9 in mice and sections of human squamous cell carcinomas. These activatable CPPs can be used to deliver not only fluorescent or contrast agents for imaging purposes, but also therapeutic molecules in a cell-specific manner. (Proc. Natl. Acad. Sci. USA 101, 1786717872, 2004)

\section{Bugging nanocrystals}

Escherichia coli has now added nanocrystal manufacture to its bag of tricks. Sweeney et al. have shown that under the right conditions this workhorse of biotechnology produces semiconductor cadmium sulfide nanocrystals that are $2-5 \mathrm{~nm}$ in size. The nanocrystals spontaneously formed in $E$. coli when it was incubated with cadmium chloride and sodium sulfide. Two parameters were critical for success. Nanocrystal formation depended on the growth phase; in electron micrographs, nanocrystals were observed at 20 times higher density in the stationary phase than in the late logarithmic phase, and were undetectable in the mid-logarithmic phase. Formation also depended on the strain of E. coli; crystals appeared in strains ABLC C and TG1, but not in R189 or DH10B. Nanocrystals have been made in filamentous fungi and yeast, but never before in bacteria. Although the dependence on growth phase and strain are not yet understood, various organic molecules, such as amino acids, fatty acids and polyphosphates, are known to promote the formation of inorganic nanocrystals. Genetic analysis of E. coli strains may reveal ways to control the nanocrystals' composition, size and shape. (Chem. Biol. 11, 1553-1559, 2004)

\section{Heart elixir}

Srivastava and colleagues have applied insights from developmental biology to develop a new strategy for mitigating the effects of a heart attack. The heart is famously ill-equipped to regenerate itself after injury, and survivors of heart attack often face long-term deficits in cardiac function. The authors focused on thymosin $\beta 4$, a G-actinbinding protein known to be strongly expressed in the embryonic mouse heart. Experiments in cells and embryonic heart explants showed that thymosin $\beta 4$ increases the migration, survival and beating rate of cardiomyocytes, effects that appear to be mediated by the AKT signaling pathway. The authors then delivered thymosin $\beta 4$ to mice either systemically or locally immediately after induction of myocardial infarction by coronary artery ligation. The treatment significantly improved several measures of cardiac function, including cardiac contraction, fraction of blood ejected from the left ventricle, size of the scar, and cardiomyocyte death within the first 24 hours after ligation. The authors speculate that thymosin $\beta 4$ or a small-molecule mimic of this protein may protect patients with heart injury. (Nature 432, 466-472, 2004) 